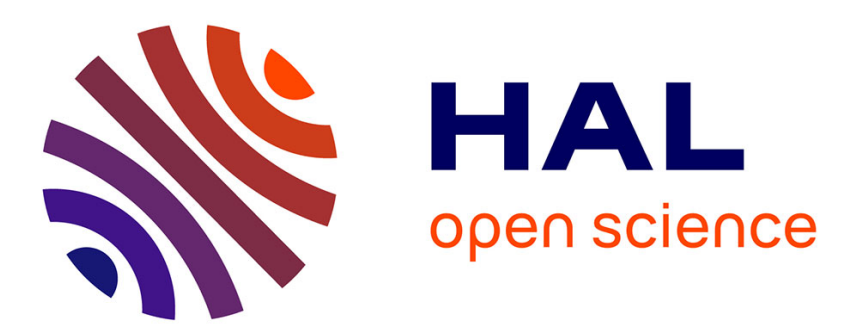

\title{
Simulating an extendable tangible slider for eyes-free one-handed interaction on mobile devices
} Juan Rosso, Céline Coutrix, Matt Jones, Laurence Nigay

\section{To cite this version:}

Juan Rosso, Céline Coutrix, Matt Jones, Laurence Nigay. Simulating an extendable tangible slider for eyes-free one-handed interaction on mobile devices. AVI '18: 2018 International Conference on Advanced Visual Interfaces, May 2018, Castiglione della Pescaia Grosseto Italy, France. pp.1-9, 10.1145/3206505.3206510 . hal-03014603

\section{HAL Id: hal-03014603 https://hal.science/hal-03014603}

Submitted on 19 Nov 2020

HAL is a multi-disciplinary open access archive for the deposit and dissemination of scientific research documents, whether they are published or not. The documents may come from teaching and research institutions in France or abroad, or from public or private research centers.
L'archive ouverte pluridisciplinaire HAL, est destinée au dépôt et à la diffusion de documents scientifiques de niveau recherche, publiés ou non, émanant des établissements d'enseignement et de recherche français ou étrangers, des laboratoires publics ou privés. 


\title{
Simulating an Extendable Tangible Slider for Eyes-Free One-Handed Interaction on Mobile Devices
}

\author{
Juan Rosso ${ }^{1}$, Céline Coutrix ${ }^{1,2}$, Matt Jones ${ }^{3}$, Laurence Nigay ${ }^{1}$ \\ ${ }^{1}$ University Grenoble Alpes, CNRS, \\ Grenoble INP, LIG \\ 38000, Grenoble, France \\ ${ }^{2}$ University of Stuttgart \\ 70569, Stuttgart, Germany \\ celine.coutrix@vis.uni-stuttgart.de
${ }^{3}$ FIT Lab, Swansea University
SA28PP, Swansea, UK
matt.jones@swansea.ac.uk

fuan-pablo.rosso, celine.coutrix, laurence.nigay\}@imag.fr

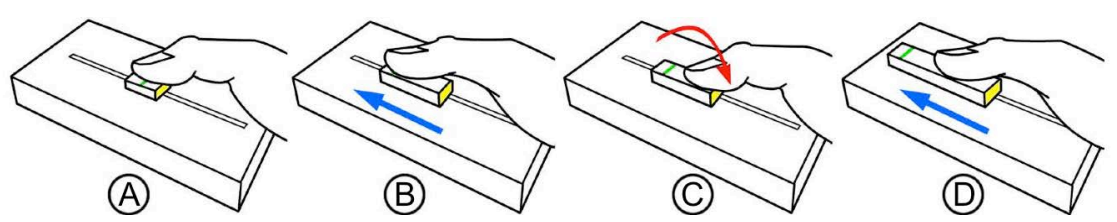

Figure 1. Clutching with an extendable tangible slider: (A) the user places her thumb on the slider knob and (B) begins to push upwards (direction of the blue arrow). (C) When this action is no longer comfortable the user is able to continue to adjust the controlled parameter (as visualized with the green landmark line) by carrying out a clutching action, drawing the thumb down to the comfortable starting position. (D) This is possible because the slider knob expands (yellow side) in the opposite direction.

\begin{abstract}
Sliders are widely used on mobile devices. Envisioning mobile devices that can dynamically deform to raise tangible controls from the screen surface, tangible sliders offer the benefit of eyesfree interaction. However, reaching for distant values with one hand is problematic: users namely need to change their handgrip, which is not comfortable. To overcome this problem, this paper sets out to experimentally study an extendable tangible slider to support one-handed clutching. The tangible slider's knob extends to maintain the thumb's movement within its comfortable area. We first built a low-fidelity prototype made of a knob long enough to allow clutching. This low-fidelity prototype significantly improves performance when reaching distant targets, as compared to a standard tangible slider. We then built a higher-fidelity prototype, introducing actuation and allowing for a shorter knob. When used for clutching, the knob moves back towards the users' thumb. Experimental results show that the motion of the actuated knob does not interrupt eyes-free interaction during manipulation. In comparison, a graphical extendable slider performed $0.9 \mathrm{~s}$ slower due to the required visual attention. However, the results suggest that the motion of the actuated knob affects performance, as the higherfidelity prototype performed $0.6 \mathrm{~s}$ slower than the low-fidelity prototype.

Permission to make digital or hard copies of all or part of this work for personal or classroom use is granted without fee provided that copies are not made or distributed for profit or commercial advantage and that copies bear this notice and the full citation on the first page. Copyrights for components of this work owned by others than ACM must be honored. Abstracting with credit is permitted. To copy otherwise, or republish, to post on servers or to redistribute to lists, requires prior specific permission and/or a fee. Request permissions from Permissions@acm.org.
\end{abstract}

AVI '18, May 29-June 1, 2018, Castiglione della Pescaia, Italy (C) 2018 Association for Computing Machinery.

ACM ISBN 978-1-4503-5616-9/18/05...\$15.00

https://doi.org/10.1145/3206505.3206510

\section{CCS CONCEPTS}

- Human-centered computing Interaction techniques

- Human-centered computing Mobile devices

\section{KEYWORDS}

Extendable slider, Mobility, Thumb interaction, Tangible interaction, Shape-changing interfaces

\section{INTRODUCTION}

Adjusting continuous parameters in an eyes-free manner is a common task. Users often need to define a parameter value within a large continuous interval. For instance, mobile audio engineers fixing the sound volume of a venue before a concert [16], scientists filtering data displayed on a wall-sized display [13], a person at home adjusting the TV sound volume while going to open the door. Current technologies fail to cover the users' demands when performing such tasks. On the one hand, standard remote controls offer discrete buttons, which can be tedious and/or difficult to operate. Repeatedly pressing a button for specifying a particular value is tiring [30]. Maintaining a button pressed and releasing it when the target is reached requires high sensorimotor coordination [3]. On the other hand, solutions on smartphones and tablets provide graphical widgets used for continuous adjustment -e.g., sliders. However, these solutions lack tactile feedback, forcing the user to visually focus on the input device to operate it. This visual dependency prevents the users from eyes-free interaction, which is a feature desired by users of mobile devices [34].

Another problem related to interaction with mobile devices is the unreachability of some areas by the thumb when using only one hand. This is particularly problematic for large sized phones, which are difficult to use in a one-handed manner [4]. This 
problem has motivated several studies on the functional area of the thumb $[2,12,15]$. When reaching outside the functional area of the thumb, users perform different types of hand movements in order to change their handgrip $[4,8,12]$. These hand movements can lead to uncomfortable handgrips for the user.

Inspired by early attempts to provide eyes-free interaction through tangibility $[11,13,20]$, a study on interacting with mobile tangible controls, focused on shrinking the tangible control to avoid handgrip changes [21]. However, the study confirmed previous work [5] by showing the negative impact of small motor scale on performance.

A solution would be to use a tangible isometric device to control the direction and speed of movement within the comfortable area, rather than the position of the cursor. However, position control was found to offer a high degree of perceived usability as compared to rate control [33]. As a consequence, in this paper we adopt another solution by considering an extendable slider capable of extending its knob length (Figure 1). The slider's knob increases its length in order to support thumb-clutching movements. When the knob is moved upwards, the knob deforms downwards while letting the slider's landmark move upwards (see Figure 1 A-B and C-D), and vice versa. Furthermore, clutching allows us to provide a large motor scale [5] while maintaining thumb's movements within its comfortable area. Users can then always manipulate the knob precisely, eyes-free, and comfortably -i.e., from the functional area of the thumb.

As a practical example of use, we consider the audio engineers' case presented in [16]. These professionals would benefit from our approach: mobility is key for audio engineers in order to check the sound at different locations around the venue. Moreover, eyes-free interaction is required to control audio parameters while looking at the stage. Finally, one-handed interaction is required while changing audio parameters - audio engineers communicate with musicians through a microphone or hand signals.

After showing how prior work on one-handed interaction contributed to our problem, we present our first user study: we measured the expected performance through a low-fidelity prototype having a very large knob. We then present our evaluation of a higher fidelity prototype that introduces actuated motion of the knob, before concluding.

\section{RELATED WORK}

In mobile situations, users most likely use one hand. An observational study, based on data from 780 people [12], revealed that there are three basic ways of how users hold their mobile phones while interacting with them: $49 \%$ interacted with the same hand that holds the phone, $36 \%$ interacted with one hand while holding the device with the other hand and $15 \%$ interacted with both hands.

The preference and dominance for one-handed interaction on mobile devices have motivated several studies on the functional area of the thumb. An empirical study by Karlson et al. [15] demonstrated that areas difficult to reach with the thumb provoke a significant slowdown (between $7 \%$ and 12\%) in movement time.

Hand movements on mobile devices have been observed in situations where users reach for targets beyond the functional area of the thumb $[4,8,12]$. When targets are near the functional area, two small movements have been identified: (1) fingers placed on the back of the device change position, and (2) the mobile device is tilted within the user's hand. We will refer to these movements as a handgrip change.

When handgrip changes are not sufficient to reach a target, a shifting of the device within the hand is observable [12]. We will refer to the shift of the device as a hand relocation.

Handgrip changes and hand relocations can be uncomfortable, unstable, and cause the falling of the device. The distinction between handgrip changes and hand relocations is important as they represent two distinct areas outside the functional area of the thumb [12]. However, the limits between these areas are not defined.

\subsection{Accessing Targets Outside the Functional Area: Graphical Interaction}

Graphical interaction techniques have been proposed by manufacturers to access targets outwith the functional area of the thumb, e.g., double-tapping the home button on iOS will bring the top half of the screen down to the thumb.

Researchers have further studied graphical interaction techniques, first leveraging the thumb. ThumbSpace [14] generates a proxy view of a part of the screen within the functional area of the thumb. The proxy view facilitates the access to out-of-reach targets with the thumb. MagStick [23] is a "magnetized" cursor allowing access to out-of-reach targets. It can be controlled from within the functional area of the thumb through a telescopic stick. Yu et al. [35] presented two techniques: the first technique is similar to Thumbspace [14] but initiated by one single swipe gesture from the thumb. The second technique makes use of a graphical circular widget that appears inside the functional area of the thumb. The widget is composed of four buttons, which allow the user to access "magnetized" elements near the corners of the screen.

Other graphical techniques consist of expanding the interaction space of the thumb by using the back of the device. Hakoda et al. [10] investigated the index finger's range on the back of the device while interacting with the thumb on the front. Löchtefeld et al. [18] presented a hybrid approach combining front- and back-of-device touch input. While the thumb is used to select reachable areas on the lower part of the display, the index finger is used on the back to reach the top left corner of the display.

\subsection{Accessing Targets Outside the Functional Area: Towards Tangible Interaction}

So far, all the studies related to the functional area of the thumb have been done for graphical interfaces. Yet, the benefits of tangibility for eyes-free interaction have been demonstrated for mobile devices, through tangible controls placed by the user on 
the display [13] and tangible controls emerging from a deformable display [20]. However, performance was not analyzed for one-handed interaction with such tangible controls $[13,20]$.

Many continuous tangible controls can be considered for eyes-free mobile interaction. Scrolling wheels lack a landmark to provide observability of the parameter. Knobs can have a landmark, but the left/right adjustment is not easily mapped to min-max of a parameter, as different cultures can differ on the spatial representation of numbers [25]. The landmark is important when the user needs to acquire the current value (through vision or touch) after the task was interrupted. In this paper, we focus on linear controls, such as vertical sliders, since they are commonly used to control a variety of functionalities e.g., sound, heat, light, TV channels, and data filtering. This allows us to ensure better adoption by providing retrocompatibility [6].

In previous work we analyzed the impact that the slider's length and orientation have on performance when used with one hand [21]. We found that shrinking the slider's length to fit it within the thumb's comfortable area has a negative impact on performance in comparison with a long slider. This is in line with the results by Chapuis and Dragicevic about the impact of reducing the motor scale (i.e., reducing the target's size in the motor space) [5] on performance.

While we adopt a similar approach by considering an emergeable tangible slider for eyes-free interaction, our aim is to design and study an extendable tangible slider that allows thumb clutching in order to support a large motor scale while maintaining the thumb's movements within its comfortable area.

\section{EXPERIMENT: BALANCING HANDGRIP CHANGES, HAND RELOCATIONS, AND CLUTCHING}

We first explore the impact that thumb-clutching movements have on performance. For this, we compared thumb-clutching, performed on a large knob, with handgrip changes and hand relocations, caused by standard tangible sliders. A withinsubjects design was used with three independent variables: Knob, Distance, and Width.

The Knob is used on a mobile device to control a graphical cursor displayed on a distant screen. The Knob variable is composed of two conditions (see Figure 2):

Small $(20 \mathrm{~mm} \times 10 \mathrm{~mm} \times 23 \mathrm{~mm})$ : To reach a target near (resp. outside) the functional area, users have to change their handgrip (resp. relocate their hand, see Figure 2A-B). It resembles a standard tangible slider;

Large $(150 \mathrm{~mm} \times 10 \mathrm{~mm} \times 23 \mathrm{~mm})$ : To reach a target outside the functional area, users perform thumb-clutching within the area (see Figure 2C). This is meant as a low-fidelity prototype of an extendable slider.

The Distance variable refers to the distance between targets displayed on a distant screen. To explore targets that force handgrip changes and hand relocations, we chose the following values: First, $116 \mathrm{~mm}$ between consecutive on-screen targets (i.e., $90 \mathrm{~mm}$ in the control space on the mobile device) proved to force handgrip changes [21]. Second, $217 \mathrm{~mm}$ between consecutive onscreen targets (i.e., $150 \mathrm{~mm}$ in the control space on the mobile device) experimentally proved to force the relocation of the hand. Both distances fit within modern large-sized mobile phones [1].

The Width variable represents the target's width. In order to analyze performance from coarse to fine adjustment, two different widths were chosen: $7 \mathrm{~mm}$ and $1.6 \mathrm{~mm}$.

The Distance $\times$ Width variables were fully crossed, defining the following task IDs $=4.2,5,6.3,7$, as computed in [26]. This confirmed our choice by giving medium and hard difficulty levels and avoiding the task being too difficult [26].

We then hypothesize that:

H1: When pointing at closest targets, thumb-clutching (large knob) and handgrip changing (small knob) perform equally well.

$\mathrm{H} 2$ : When pointing at farthest targets, thumb-clutching (large knob) outperforms hand relocations (small knob).

H3: When pointing at smallest targets, thumb-clutching (large knob) outperforms both handgrip changes and hand relocations (small knob).

\subsection{Apparatus and Participants}

A single body for the prototype (see Figure 2) was built with the following dimensions: $150 \mathrm{~mm}$ (height) $\times 70 \mathrm{~mm}$ (width) $\times 17 \mathrm{~mm}$ (thickness), giving a total thickness of $40 \mathrm{~mm}$ when using the knobs. An exploratory study [22] underlined the impact of the device's thickness on performance. In this study, we controlled the thickness of both knobs to be the same. The length and width were the ones of a commercial large phone [1] for a realistic grip. The prototype was built using a $3 \mathrm{~mm}$-thick lasercut medium-density fiberboard. A membrane potentiometer [27] of $200 \mathrm{~mm}$ length captures the position of the slider's cursor. The slider and the knobs were made with an Ultimaker 2+ 3D printer, using PLA as the filament. The potentiometer was connected to an Arduino Mega 2560 board. The board was connected via USB

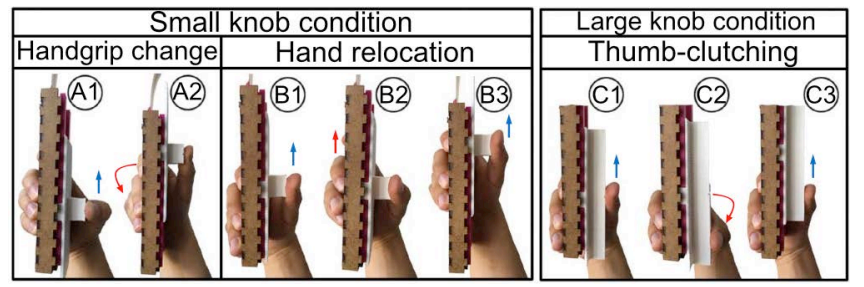

Figure 2. The Knob conditions and the hand movements related to their operation: (A1) the small knob is pushed (direction of the blue arrow) with an initial handgrip. (A2) The fingers on the back of the device move (direction of the red arrow), thus changing the handgrip.

(B1) the small knob is pushed (direction of the blue arrow) with an initial hand location. (B2) The hand is relocated (direction of the red arrow) when the thumb's limit is reached. (B3) The knob is pushed with the new hand location.

(C1) The large knob is pushed (direction of the blue arrow) with an initial handgrip. (C2) Relocation of the thumb. (C3) The knob is pushed with the same handgrip. 
to a MacBook Pro running the experimental software, displayed on a 27 -inch Thunderbolt screen $(2560 \times 1440,109$ ppi).

Sixteen volunteers (between 23 and 34 years old, $M=27.3,11$ males and 5 females) were recruited on campus. All were righthanded and owners of touchscreen phones. The average measured distance between participants' thumb and index finger with the open hand was $176 \mathrm{~mm}(\mathrm{SD}=12.1 \mathrm{~mm})$.

\subsection{Task}

The study requires participants to perform a distant pointing task, as in several previous studies e.g., [7, 20,23]. This abstract task allows representing of real-life tasks in which users adjust a distant parameter without looking at the mobile input device, e.g., browsing a list of channels displayed on the TV, fixing the sound volume while looking at a stage.

The experimental task (see Figure 3) consisted of the user controlling the input device without looking at it. The visual focus was on a screen placed at a distance of $1.60 \mathrm{~m}$. The mobile device controlled the cursor of a graphical slider displayed on the distant screen. The graphical slider had a length of $232 \mathrm{~mm}$. The cursor, controlled by the user, was displayed as a white horizontal line (Figure 3). A visual feedback of the remaining distance (in red) from the user's cursor to the target (in green) is displayed along the slider. Participants were asked to point as fast as possible.

For validation, users were asked to maintain the cursor in the target area for 1s. This validation mechanism is used to avoid any additional error-prone actions [28]. The extra second was subtracted from the movement time before analysis. Overshoots (i.e., passing over a target - entering and leaving the target area) are used to indicate pointing accuracy [28]. After the task is completed successfully, a new target appears at a predefined Distance $(116 \mathrm{~mm}$ or $217 \mathrm{~mm})$ from the current location of the user's cursor.

\subsection{Dependent Variables}

Our main objective in this study was to determine the impact of thumb-clutching (large knob), handgrip changes and hand relocations (small knob) on performance. To this end, we recorded the movement time from the beginning of each task

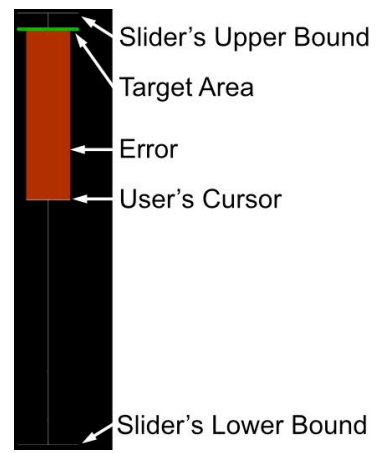

Figure 3. Screenshot of the experimental pointing task with a slider of $232 \mathrm{~mm} / 1121 \mathrm{px}$, a target's width of $1.6 \mathrm{~mm} / 7 \mathrm{px}$, and a distance of $217 \mathrm{~mm} / 1048 \mathrm{px}$. until the validation. In addition, we recorded the number of performed overshoots in order to study the accuracy of the hand movements (as done in [28]). Finally, as an indication of perceived usability of the tested knobs, we asked them to fill in a System Usability Scale (SUS) form [31]. The actions of the participants were also video-recorded to study the required hand movements to operate the prototypes (i.e., handgrip changes and hand relocations for the small knob).

\subsection{Procedure}

Participants were first introduced to the prototype through a training phase in which they performed the tasks with the different combinations Knob $\times$ Distance $\times$ Width. During training, the order of presentation of the conditions was randomized. The training phase lasted 10 minutes on average. Afterwards, the trials started.

The tasks were performed in 2 blocks, one per Knob condition. Half of the participants started with the large knob and continued with the small one and vice versa for the other participants. Each block was divided into 4 sub-blocks representing the 4 Distance $\times$ Width combinations. The presentation order of the sub-blocks was randomized. For each sub-block, participants performed 17 repetitions of the task. The first repetition was not analyzed to avoid having results affected by the knob's position from the previous sub-block. A small break was allowed after each sub-block. After participants completed the task for the 2 blocks, they were given the SUS form to fill in.

A total of 2048 measures of movement time were collected, from 16 participants $\times 16$ repetitions $\times 2$ knobs $\times 2$ distances between targets $\times 2$ target's widths. This resulted in 256 measures for each Knob $\times$ Distance $\times$ Width condition.

\subsection{Results}

We used the geometric mean to estimate the center of the distribution of movement times [24]. Trials were aggregated using the factors Knob, Distance, and Width for each participant. A Shapiro-Wilk test revealed that we could not assume the normality of the data $(W=0.9, p<0.001)$. Thus, we applied an Aligned Rank Transformation on the data [32] and then ran repeated measures ANOVA on the aligned ranks to investigate possible interactions between factors.

A three-way ANOVA shows that $\operatorname{Knob}(F(1,105)=32.9, p<$ $0.0001)$, Distance $(F(1,105)=191.9, p<0.0001)$ and Width $(F(1$, $105)=634.4, p<0.0001)$ had a significant impact on movement time (Figure 4). An interaction between Width and Distance $(\mathrm{F}(1$, $105)=4.8, \mathrm{p}<0.05)$ proved to be significant. This confirms that the Width values were appropriate to distinguish between fine and coarse adjustment for the Distance values.

An interaction between Knob and Distance $(\mathrm{F}(1,105)=8.5, \mathrm{p}<$ 0.01 ) proved to be significant. For the $217 \mathrm{~mm}$ Distance condition, the large Knob performed faster for both $1.6 \mathrm{~mm}$ and $7 \mathrm{~mm}$ target's Width ( $+0.6 \mathrm{~s}$ and $+0.4 \mathrm{~s}$ respectively) than the small Knob. A Tukey's post-hoc test revealed a significant difference between the large and small Knob conditions $(t(105)=5,7, p<0.001$, 


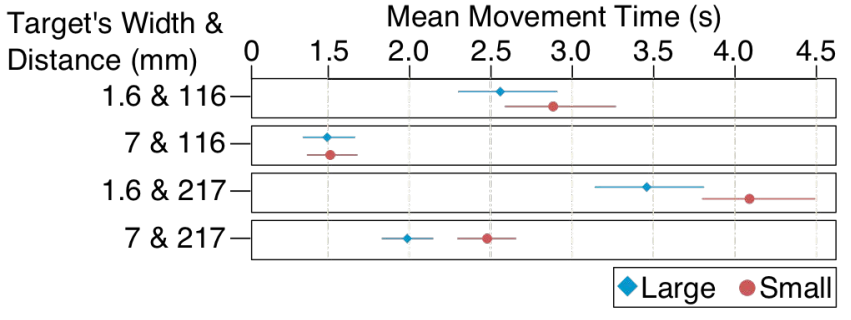

Figure 4. Mean movement time for the 2 Knob conditions and for the 4 possible Distance $\times$ Width conditions ( $y$-axis). Error bars show $95 \%$ confidence intervals.

Cohen's $d=0.5)$. For the $116 \mathrm{~mm}$ Distance condition, the large Knob performed better $(+0.4 s)$ than the small Knob for a target's Width of $1.6 \mathrm{~mm}$. For the $7 \mathrm{~mm}$ target's Width, the large and small Knobs performed equally. We illustrate this in Figure 4.

To explain the equal performance of the Knob conditions for the single $7 \mathrm{~mm} \times 116 \mathrm{~mm}$ condition, we used the video footage. We compared the amount of thumb-clutching and handgrip changes performed during the task. On average, 1 clutching movement on the large Knob was required $(\mathrm{SD}=0.4)$. Only 1 handgrip change was required on the small Knob. This suggests that when reaching large targets with a short distance between targets, a small knob perform equally well as a large knob.

Regarding the number of overshoots, on average, for large targets $(7 \mathrm{~mm})$ and long distances $(217 \mathrm{~mm})$, participants overshot two times $(\mathrm{SD}=0.02)$ with the large knob; and 4 times $(\mathrm{SD}=0.03)$ with the small knob. For large targets and short distances $(116 \mathrm{~mm})$, participants overshot 3 times $(\mathrm{SD}=0.03)$ with the large knob; and 4 times $(\mathrm{SD}=0.04)$ with the small knob. For small targets $(1.6 \mathrm{~mm})$ and long distances $(217 \mathrm{~mm})$, participants overshot 13 times $(\mathrm{SD}=0.09)$ with the large knob; and 17 times $(\mathrm{SD}=0.13)$ with the small knob. For small targets and short distances, participants overshot 15 times $(\mathrm{SD}=0.13)$ with the large knob; and 12 times $(\mathrm{SD}=0.09)$ with the small knob. This suggests that thumb-clutching movements are slightly more precise than hand relocations and handgrip changes for: 1) large targets for both distances between targets, and 2) small targets and long distances. For small targets and short distances, handgrip changes are slightly more precise.

Contrastingly, SUS indicates that the small knob (69/100, 51\% when normalized [31]) is perceived slightly more usable than the large knob (65/100, 48\%).

\subsection{Discussion}

In this section, we discuss the observed effects that had an impact on the performance of the hand movements engaged in the Knob conditions.

\subsubsection{Short Distances: Thumb-clutching vs. Handgrip Changes}

For small targets $(1.6 \mathrm{~mm})$ and large targets $(7 \mathrm{~mm})$ near the functional area of the thumb $(116 \mathrm{~mm})$, thumb-clutching movements done with the large knob outperform handgrip changes done with the small knob $(+0.4 s)$. Moreover, participants were also more precise with thumb-clutching. Participants overshot 9 times more on average with the small knob than with the large one. $H 1$ is consequently discarded.

\subsubsection{Long Distances: Thumb-clutching vs. Hand Relocations}

Similarly to short distances, for small targets $(1.6 \mathrm{~mm})$ outside the functional area of the thumb $(217 \mathrm{~mm})$, thumb-clutching movements done with the large knob outperform hand relocations done with the small knob $(+0.6 s)$. Participants overshot 9 times more on average with the small knob than with the large knob. This result suggests again greater precision with the large knob than with the small knob.

Regarding large targets $(7 \mathrm{~mm})$, thumb-clutching outperformed hand relocations $(+0.5 \mathrm{~s})$. This suggests that hand relocations are more time-consuming than a thumb-clutching. We argue that it is caused by the special care needed to relocate the hand without dropping the device: video footage shows that participants used all the fingers to displace the device.

Overall, hand relocations were slower and less precise than thumb-clutching when pointing outside the functional area of the thumb; supporting $H 2$.

The aforementioned results on thumb-clutching movements support the results about clutching on relative pointing devices [19].

\subsubsection{Small Targets}

Thumb-clutching movements outperformed handgrip changes and hand relocations when reaching small targets $(1.6 \mathrm{~mm})$ for both short $(116 \mathrm{~mm})$ and long $(217 \mathrm{~mm})$ distances. As explained before, thumb-clutching enabled participants to perform faster and more precise, thus to reduce overshoot; supporting $H 3$.

\subsubsection{Preference}

Participants found the small knob slightly more usable, presumably due to the similar operability between the small knob and graphical sliders, and the familiarity with the latter. However, they also reported fatigue after using the small knob. This is confirmed by the video footage: participants were shaking their hands to relax their muscles during the breaks. Finally, participants indicated that the large knob was more comfortable to use since they only needed to move their thumbs.

\section{EXPERIMENT WITH AN ACTUATED SLIDER}

Thumb-clutching proved to perform well for targets at the border and far from the functional area of the thumb while offering a stable handgrip. However, in this solution the knob is long. Even on a future miniature prototype, the knob has to be long in order to allow clutching. Unfortunately, a large knob hinders portability of the solution.

We, therefore, explore a solution with a smaller knob. For this, we introduce actuation: we build a prototype that actuates the location of a small knob so that it moves back in the functional area of the thumb after clutching.

We experimentally evaluate this prototype. We were particularly interested in observing if the motion of the actuated 

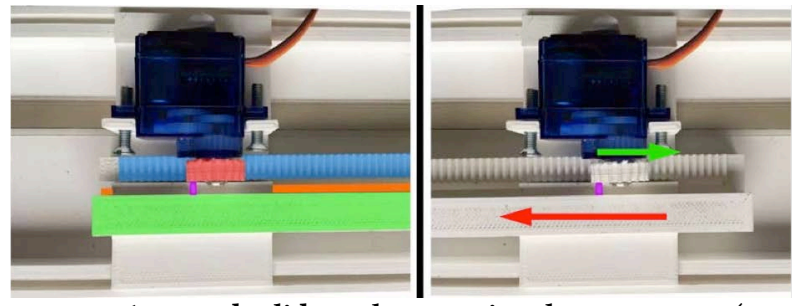

Figure 5. Actuated slider: the rotational movement (green arrow) of the pinion (in red) is transferred to a bidirectional movement (red arrow) on the rack (in blue), provoking the solid block (in orange) slide through the base and thus, simulating the extension of the knob (in green). A physical landmark (in purple) represents the position of the specified value.

knob disrupts the users from being eyes-free. In addition, we wanted to study the impact that the motion of the actuated knob has on the performance of thumb-clutching movements. Finally, we analyzed the perceived usability of such a device.

\subsection{Actuated Prototype}

The starting point is to automatically slide the knob of the slider back to the functional area of the thumb. The prototype is formed by: 1) a base composed of the slider's landmark and a FEETECH FS90R [9] continuous rotation servomotor with a pinion on its top; and 2) a piece composed of a solid block, a rack, and a flat surface that serves as the slider's knob (see Figure 5). Its length is half (0.53) the length of the previous prototype.

The pinion-rack mechanism locks the movement between the base and the knob, hence, when the user pushes the knob upwards or downwards, the base moves in the same direction. This gives the sensation of directly pushing the slider's landmark. The mechanism also transforms the rotational movement of the servomotor to a bidirectional linear movement along the slider's axis. The rotation of the pinion makes the solid block slide within the base. This gives the users the feeling that the knob is long, or becoming longer upwards or downwards according to the current position of the landmark (see Figure 6).

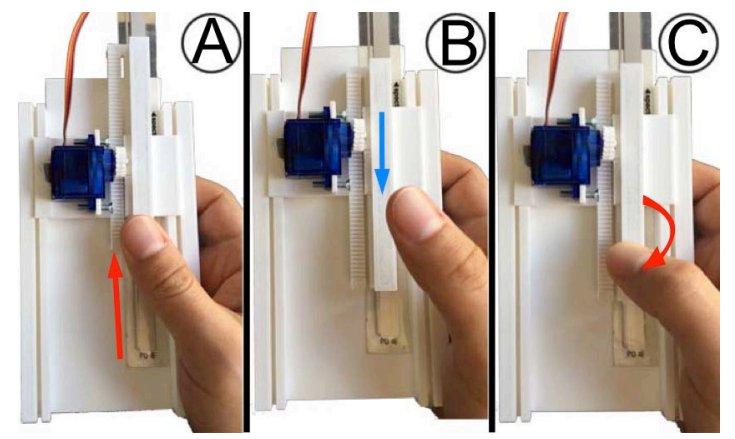

Figure 6. (A) The thumb pushes the knob (direction of the red arrow) until it reaches its maximum elongation. (B) The knob starts to move back (direction of the blue arrow) while the thumb clutches to a comfortable position. (C) The thumb is relocated within its comfortable area and ready to continue operating.
This allows the thumb to keep manipulating the slider within its comfortable area.

As on any slider, the landmark indicates the current position of the knob. We minimized the thickness to make it as portable and mobile as possible. The obtained thickness is then less than the prototype from the first study $(20 \mathrm{~mm})$. The knob is $80 \mathrm{~mm}$ long in order to remain within the thumb's comfortable area while reaching the extremes of the slider. The knob is $10 \mathrm{~mm}$ wide as for any standard slider knob.

\subsection{Comparative Experiment}

We performed the same task as in the first experiment but with the actuated knob instead of the large knob. We considered two conditions for the actuated knob in order to study eyes-free interaction with it: one condition for which the participant could look at the mobile device to receive visual feedback and one condition with no possible visual feedback by covering the input device with an opaque plastic bag. We also compared these two conditions with an extendable graphical slider. The goal was to compare the performance of clutching when manipulating: 1) an actuated knob that requires no visual attention and, 2) a graphical extendable one that requires switching the visual attention from the mobile device to a distant display.

We thus considered a Technique variable composed of three conditions. The following three conditions allow participants to perform thumb-clutching movements within the functional area of the thumb with a stable handgrip:

Tangible slider with visual feedback (Tangible-Visual): Participants are free to look at the input device and obtain visual feedback from the physical landmark (see Figure 7A).

Tangible slider with no visual feedback (Tangible-Blinded): Same as Tangible-Visual but the tangible prototype is hidden, thus preventing visual feedback as in [17]. Participants were asked to operate the prototype inside an opaque plastic bag (see Figure 7B).

Graphical slider with visual feedback (Graphical-Visual): An extendable graphical slider that enables users to operate it in the same way as its tangible counterpart (see Figure 7C).

We discarded the condition with a graphical slider and no visual feedback after we ran a pilot study that showed that it was not possible to properly manipulate the graphical extendable slider without looking at the mobile device.

We consider the same Distance and Width conditions as in the first study for this experiment. Given these conditions, we hypothesize the following:

H1: Eyes-free interaction with an actuated knob: manipulating an actuated knob will not result in a decrease of

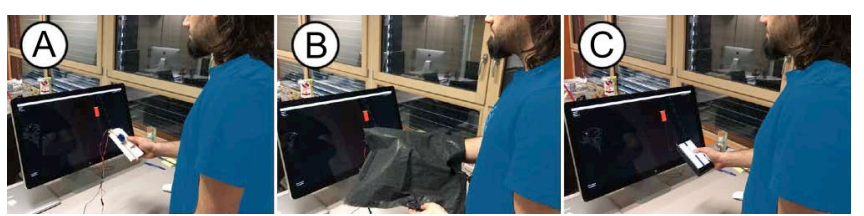

Figure 7. A participant trails under different conditions: (A) Tangible slider with visual feedback. (B) Tangible slider with no visual feedback. (C) Graphical slider with visual feedback. 
performance when users are not looking at the device. The automatic motion of the knob does not mean one has to look at the mobile input device.

H2: Manipulating an actuated knob versus switching visual attention: manipulating an actuated knob will not result in a decrease of performance compared to the equivalent graphical input method that requires switching visual attention between the mobile device and the screen.

\subsection{Apparatus and Participants}

The tangible slider was printed with the Ultimaker 3+3D printer with PLA filament. For the Graphical-Visual, an ASUS Zenfone 2 Laser (6-inch screen) smartphone [1] was used. We 3D printed a case for the smartphone in order to have the same thickness, equal to $20 \mathrm{~mm}$, between the tangible and graphical prototypes. We made use of the same experimental software and connection setup as in the first experiment to operate the tangible prototypes. For the graphical prototype, the communication with the experimental software was done through a Wi-Fi connection.

Seventeen volunteers (between 23 and 34 years old, $M=26,11$ males and 6 females) were recruited on campus.

\subsection{Task and Procedure}

For this experiment, participants performed the same task and followed the same procedure as in the first experiment.

A total of 3264 measures of movement time were collected, from 17 participants $\times 16$ repetitions $\times 3$ techniques $\times 2$ target's widths $\times 2$ distances between targets. For each Technique $\times$ Width $\times$ Distance conditions, this resulted in 272 measures of movement time.

As in the first experiment, we recorded the movement time to complete the task and the number of performed overshoots for each Technique. We also video recorded the thumb movements and gaze of participants while operating the prototypes (as done in [20]): we analyzed gaze diversions and thumb movements during the automatic motion of the actuated knob.

\subsection{Results}

As in the first experiment, we used the geometric mean to aggregate movement time. The data was aggregated per participant and variables. A Shapiro-Wilk test revealed that we could not assume the normality of the data $(\mathrm{W}=0.9, \mathrm{p}<0.01)$. Thus, we applied an Aligned Rank Transformation on the data [32].

A repeated measures three-way ANOVA showed a significant main effect of Technique $(\mathrm{F}(2,176)=62.7, \mathrm{p}<0.0001)$, Distance $(\mathrm{F}(1,176)=123.7, \mathrm{p}<0.0001)$ and Width $(\mathrm{F}(1,176)=118.7, \mathrm{p}<$ 0.0001 ) on movement time. As in the previous experiment, the differences in movement time between small $(1.6 \mathrm{~mm})$ and large targets $(7 \mathrm{~mm})$ for the same distance confirm that the chosen conditions were appropriate for distinguishing between fine and coarse adjustment (see Figure 8).

An interaction between Technique and Distance $(F(2,176)=$ $9.5, p<0.001)$ proved to be significant. For the $116 \mathrm{~mm}$ Distance condition, the Graphical-Visual condition presents the highest

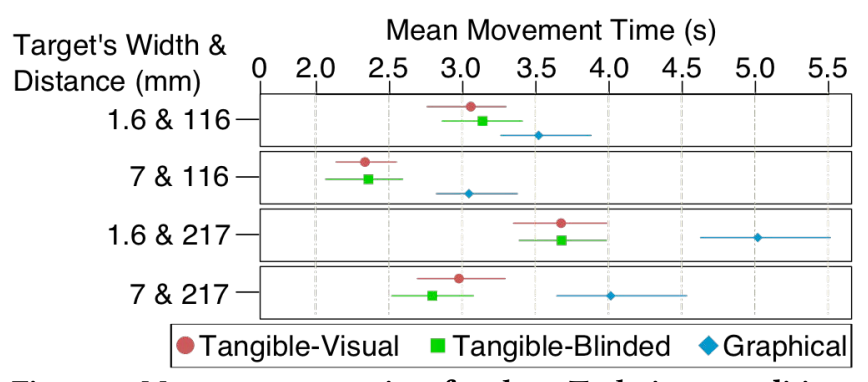

Figure 8. Mean movement time for the 3 Technique conditions and for the 4 possible Distance $\times$ Width conditions (y-axis). Error bars show $95 \%$ confidence intervals.

movement time on both $1.6 \mathrm{~mm}$ and $7 \mathrm{~mm}$ target's width $(3.5 \mathrm{~s}$ and $3 s$ respectively). A Tukey's post-hoc test revealed no significant difference between the Tangible-Visual and Tangible-Blinded conditions for both widths $(\mathrm{t}(80)=5.5, \mathrm{p}>0.05$, Cohen's $\mathrm{d}=0.4)$. Similarly, for the $217 \mathrm{~mm}$ Distance condition, the GraphicalVisual condition presented the slowest performance $(+1.1 \mathrm{~s}$ from the tangible conditions) for both target's widths. A Tukey's posthoc test revealed no significant difference on performance between the Tangible-Visual and Tangible-Blinded conditions for both target's widths $(t(80)=5.5, p>0.05$, Cohen's $d=0.2)$. These differences are observable in Figure 8 where the Tangible-Visual and Tangible-Blinded conditions have similar performance times. This suggests that, when operating tangible controls, having visual feedback from the input device is not critical for performance. On the contrary, visual attention was required to operate the Graphical-Visual condition.

Regarding the number of overshoots, on average, for short distances $(116 \mathrm{~mm})$ and small targets $(1.6 \mathrm{~mm})$, participants overshot 9.8 times $(\mathrm{SD}=0.07)$ with the Tangible-Visual condition, 14.7 times $(\mathrm{SD}=0.14)$ with the Tangible-Blinded condition, and 15 times ( $\mathrm{SD}=0.08)$ with the Graphical-Visual. For large targets $(7 \mathrm{~mm})$, participants overshot 6.4 times $(\mathrm{SD}=0.05)$ with the Tangible-Visual condition, 6.5 times $(\mathrm{SD}=0.05)$ with the TangibleBlinded condition, and 7 times $(\mathrm{SD}=0.05)$ with the GraphicalVisual. This suggests that the tangible prototype is slightly more precise than the graphical one for small and large targets over short distances between targets.

For long distances $(217 \mathrm{~mm})$ and small targets $(1.6 \mathrm{~mm})$, participants overshot 21.7 times $(\mathrm{SD}=0.17)$ with the TangibleVisual condition, 21.9 times $(\mathrm{SD}=0.15)$ with the Tangible-Blinded condition, and 14.3 times $(\mathrm{SD}=0.07)$ with the Graphical-Visual. For large targets $(7 \mathrm{~mm})$, participants overshot 7.5 times $(\mathrm{SD}=0.06)$ with the Tangible-Visual condition, 10.4 times $(\mathrm{SD}=0.08)$ with the Tangible-Blinded condition, and 4.4 times $(\mathrm{SD}=0.03)$ with the Graphical-Visual. This suggests that the graphical prototype is more precise than the tangible one for small and large targets over long distances between targets.

The SUS scores indicate that participants found both graphical slider $(72.1 / 100,53 \%$ after normalization [31]) and tangible slider $(71.7 / 100,52.7 \%)$ as equally usable.

\subsection{DISCUSSION}

In this section, we discuss the observed effects that had an impact on the performance of each technique. 


\subsubsection{Eyes-free Interaction With an Actuated Tangible Knob}

The Tangible-Visual condition performed equally well as the Tangible-Blinded condition for both short and long distances. Based on the number of overshoots, the Tangible-Visual was slightly more precise than the Tangible-Blinded for small targets and short distances ( -4 overshoots), and large targets and long distances ( -3 overshoots). These results suggest that the motion of the actuated knob did not prevent participants from operating in an eyes-free manner, thus supporting $H 1$.

On the contrary, the Graphical-Visual presented the worst performance. We explain the bad performance of the graphical solution by the number of time that participants looked down to reacquire the slider's knob. This effect has already been observed when operating graphical widgets $[13,17,20]$. We used video footage from the experiment to annotate each time participants gazed at the graphical slider on the input device. The result shows that participants looked down at the input device, on average, two times per repetition of the task; giving a total of 128 gaze deviations. We argue that the parabolic movement of the thumb [2] prevented participants from operating the slider in a vertical manner, thus leading participants to operate outside the slider area. Moreover, the lack of tangible feedback from the Graphical-Visual condition made difficult for users to land their thumb back into the slider graphical area when clutching. On the contrary, the tangible prototype under both visual conditions did not require visual attention to be operated.

\subsubsection{Impact of the Motion of the Actuated Tangible Knob on Performance}

The motion of the actuated knob had an unexpected effect on interaction: participants stopped manipulating the slider while the knob was in motion. Video footage shows that, when performing thumb-clutching movements, 15 out of 17 participants waited for the knob to stop moving before landing their thumb. Since participants stopped manipulating the slider, we argue that the motion of the knob had an impact on performance. To confirm this, we compared the mean number of times the operating hand was idle when using the actuated slider with the large knob from the first experiment. We found that the mean number of idle time increased $35 \%$ for the $1.6 \mathrm{~mm}$ Width condition and $64 \%$ for the $7 \mathrm{~mm}$ Width condition in comparison with the same conditions from the first experiment. Although both conditions were not tested in the same experiment, we expected that the thinner body of the designed actuated knob ($20 \mathrm{~mm}$ ) would have implied an easier operation, thus better performance. However, this was not the case. We can then only speculate that the motion of the actuated slider had an impact on the performance of thumb-clutching movements.

Despite the observed stops in movement provoked by the actuated knob and its resulting drop in performance $(+0.6 s)$ in comparison with the large knob from the first experiment, the tangible slider proved to outperform the graphical slider. This suggests that the switching of visual attention required by the graphical extendable slider had a bigger impact on performance than the motion of the actuated knob; supporting $\mathrm{H} 2$.

\subsubsection{Preference}

Both graphical and tangible sliders were found equally usable. We argue that despite the visual attention required by the graphical slider, its high score is due to the familiarity of the participants with tactile interfaces [29].

Regarding our tangible prototype, we argue that a higher fidelity version could have been perceived as more useful [20]. Nevertheless, we consider our tangible prototype as being of medium fidelity since it supported good performance.

\section{CONCLUSION}

In this paper, we introduced thumb-clutching on tangible extendable sliders for eyes-free and one-handed interaction on mobile devices. We built two prototypes to test the viability and performance of thumb-clutching on tangible extendable sliders.

Our first low-fidelity prototype - a tangible slider with a very large knob - gave us insight into the manipulation and performance of an extendable slider and how it compares to a standard tangible slider. Experimental results show that clutching on this prototype improves performance when reaching for proximal and distant targets.

Our second higher-fidelity prototype - a tangible slider with an actuated knob - was compared to an extendable graphical slider. The results show that (1) the motion of the actuated knob did not disrupt participants from eyes-free interaction; (2) the switching of the visual attention required to operate the graphical extendable slider has a bigger impact on performance than the stops in manipulation during the automatic motion of the actuated knob.

Our results show the benefits of thumb-clutching on tangible extendable sliders for eyes-free and one-handed interaction in the context of remote displays. To address the limitations of the designed prototype we will in the future work test different directions for deforming the knob instead of moving it. For instance, the knob could extend by morphing out of the surface below the thumb; this would prevent the user from feeling the motion of the knob and therefore stopping the manipulation of the slider. Furthermore, we will investigate the challenge of combining the use of multiple extendable tangible sliders for the adjustment of different continuous parameters -e.g., controlling the RGB levels of a projector.

\section{ACKNOWLEDGMENTS}

This work was supported by the French National Research Agency (ANR-11-LABX-0025-01, ANR-11-EQPX-0002, ANR-15CE23-0011-01), the EPSRC grant (EP/N013948/1), the Alexander von Humboldt Foundation, the German Research Foundation through projects C02 \& C04 of SFB/TRR 161, and the German Federal Ministry of Education and Research as part of the project Be-greifen (grant no. 16SV7527). The prototypes have been partially made in MSTIC (http://fabmstic.liglab.fr/). 


\section{REFERENCES}

[1] ASUS. 2017. Zenfone 2 Laser. https://www.asus.com/Phone/ZenFone-2-LaserZE500KL/. (2017). Accessed: 11-01-2018

[2] Joanna Bergstrom-Lehtovirta and Antti Oulasvirta. 2014. Modeling the functional area of the thumb on mobile touchscreen surfaces. In Proceedings of the SIGCHI Conference on Human Factors in Computing Systems (CHI '14). ACM, New York, NY, USA, 1991-2000. DOI: https://doi.org/10.1145/2556288.2557354

[3] Roald A. Bjørklund. 1991. Reaction time and movement time measured in a key-press and a key-release condition. Perceptual and Motor Skills 72, 2 (1991), 663-673. PMID: 1852576

[4] Youli Chang, Sehi L'Yi, Kyle Koh, and Jinwook Seo. 2015. Understanding Users' Touch Behavior on Large Mobile Touch-Screens and Assisted Targeting by Tilting Gesture. In Proceedings of the 33rd Annual ACM Conference on Human Factors in Computing Systems (CHI '15). ACM, New York, NY, USA, 1499-1508. DOI: https://doi.org/10.1145/2702123.2702425

[5] Olivier Chapuis and Pierre Dragicevic. 2011. Effects of motor scale, visual scale, and quantization on small target acquisition difficulty. ACM Trans. Comput.-Hum. Interact. 18, 3, Article 13 (August 2011), 32 pages. DOI: https://doi.org/10.1145/1993060.1993063

[6] Andy Cockburn, Carl Gutwin, Joey Scarr, and Sylvain Malacria. 2014 Supporting Novice to Expert Transitions in User Interfaces. ACM Comput. Surv. 47, 2, Article 31 (November 2014), 36 pages. DOI: http://dx.doi.org/10.1145/2659796

[7] Céline Coutrix and Cédric Masclet. 2015. Shape-Change for Zoomable TUIs: Opportunities and Limits of a Resizable Slider. In Human Computer Interaction - INTERACT 2015. Lecture Notes in Computer Science, Vol. 9296. Springer International Publishing, 349-366. DOI: http://dx.doi.org/10.1007/978-3-31922701-6_27

[8] Rachel Eardley, Steve Gill, Anne Roudaut, Stephen Thompson, and Joanna Hare. 2016. Investigating how the hand interacts with different mobile phones. In Proceedings of the 18th International Conference on HumanComputer Interaction with Mobile Devices and Services Adjunct (MobileHCI '16). ACM, New York, NY, USA, 698-705. DOI https://doi.org/10.1145/2957265.2961840

[9] Feetech. 2017. Micro 360 Degrees Continuous Rotation Servo http://www.feetechrc.com/product/analog-servo/micro-1-3kg-cm-360-degreecontinuous-rotation-servo-fs90r/. (2017). Accessed: 11-01-2018

[10] Hiroyuki Hakoda, Yoshitomo Fukatsu, Buntarou Shizuki, and Jiro Tanaka. 2015. Back-of-Device Interaction based on the Range of Motion of the Index Finger. In Proceedings of the Annual Meeting of the Australian Special Interest Group for Computer Human Interaction (OzCHI '15), Bernd Ploderer, Marcus Carter, Martin Gibbs, Wally Smith, and Frank Vetere (Eds.). ACM, New York, NY, USA, 202-206. DOI: https://doi.org/10.1145/2838739.2838812

[11] Fabian Hemmert, Gesche Joost, André Knörig, and Reto Wettach. 2008. Dynamic knobs: shape change as a means of interaction on a mobile phone. In CHI '08 Extended Abstracts on Human Factors in Computing Systems (CHI EA '08). ACM, New York, NY, USA, 2309-2314. DOI https://doi.org/10.1145/1358628.1358675

[12] Steven Hoober. 2011. How Do Users Really Hold Mobile Devices? http://www.uxmatters.com/mt/archives/2013/02/how-do-users-really-holdmobile-devices.php. (2011). Accessed: 11-01-2018

[13] Yvonne Jansen, Pierre Dragicevic, and Jean-Daniel Fekete. 2012. Tangible remote controllers for wall-size displays. In Proceedings of the SIGCHI Conference on Human Factors in Computing Systems (CHI '12). ACM, New York, NY, USA, 2865-2874. DOI: http://dx.doi.org/10.1145/2207676.2208691

[14] Amy K. Karlson and Benjamin B. Bederson. 2007. ThumbSpace: generalized one-handed input for touchscreen-based mobile devices. In Proceedings of the 11th IFIP TC 13 international conference on Human-computer interaction (INTERACT'07), Cécilia Baranauskas, Philippe Palanque, Julio Abascal, and Simone Diniz Junqueira Barbosa (Eds.). Springer-Verlag, Berlin, Heidelberg, 324-338

[15] Amy K. Karlson, Benjamin B. Bederson, and Jose L. Contreras-Vidal. 2008. Understanding One-Handed Use of Mobile Devices. In Handbook of Research on User Interface Design and Evaluation for Mobile Technology. IGI Global, Hershey, PA, USA, Chapter 6, 86--101. DOI: http://dx.doi.org/10.4018/978-159904-871-0.ch006

[16] Hyunyoung Kim, Céline Coutrix and Anne Roudaut. 2018 KnobSlider: Design of a Shape-Changing UI for Parameter Control. In Proceedings of the SIGCHI Conference on Human Factors in Computing Systems (CHI'18), April 21-26, Montréal, Canada, ACM. 10+3 pages. DOI: https://doi.org/10.1145/3173574.3173913

[17] Lars Lischke, Paweł W. Woźniak, Sven Mayer, Andreas Preikschat, and Morten Fjeld. 2017. Using Variable Movement Resistance Sliders for Remote Discrete Input. In Proceedings of the 2017 ACM International Conference on Interactive Surfaces and Spaces (ISS '17). ACM, New York, NY, USA, 116-125. DOI: https://doi.org/10.1145/3132272.3134135

[18] Markus Löchtefeld, Christoph Hirtz, and Sven Gehring. 2013. Evaluation of
Hybrid Front- and Back-of-device Interaction on Mobile Devices. In Proceedings of the 12th International Conference on Mobile and Ubiquitous Multimedia (MUM '13). ACM, New York, NY, USA, Article 17, 4 pages. DOI: http://dx.doi.org/10.1145/2541831.2541865

[19] Mathieu Nancel, Daniel Vogel, and Edward Lank. 2015. Clutching Is Not (Necessarily) the Enemy. In Proceedings of the 33rd Annual ACM Conference on Human Factors in Computing Systems (CHI '15). ACM, New York, NY, USA, 4199-4202. DOI: http://dx.doi.org/10.1145/2702123.2702134

[20] Simon Robinson, Céline Coutrix, Jennifer Pearson, Juan Rosso, Matheus Fernandes Torquato, Laurence Nigay, and Matt Jones. 2016. Emergeables: Deformable Displays for Continuous Eyes-Free Mobile Interaction. In Proceedings of the 2016 CHI Conference on Human Factors in Computing Systems (CHI '16). ACM, New York, NY, USA, 3793-3805. DOI: https://doi.org/10.1145/2858036.2858097

[21] Juan Rosso, Céline Coutrix, Matt Jones, and Laurence Nigay. 2016. Impact of mobile tangible slider design and its reachability on pointing performance. In Actes de la 28ième conférence francophone sur l'Interaction HommeMachine (IHM '16). ACM, New York, NY, USA, 70-78. DOI: https://doi.org/10.1145/3004107.3004123

[22] Juan Rosso, Céline Coutrix, Matt Jones, and Laurence Nigay. 2017. Deformable Tangible Slider for Eyes-Free One-Handed Thumb Interaction on Mobile Devices. In Actes de la 29ième conférence francophone sur l'Interaction HommeMachine (IHM '17). AFIHM, Ed., AFIHM, ACM (Poitiers, France, Aug. 2017), 11 p. DOI: http://dx.doi.org/10.1145/3132129.3132134

[23] Anne Roudaut, Stéphane Huot, and Eric Lecolinet. 2008. TapTap and MagStick: improving one-handed target acquisition on small touch-screens. In Proceedings of the working conference on Advanced visual interfaces (AVI '08). ACM, New York, NY, USA, 146-153. DOI: https://doi.org/10.1145/1385569.1385594

[24] Jeff Sauro and James R. Lewis. 2010. Average Task Times in Usability Tests: What to Report?. In Proceedings of the SIGCHI Conference on Human Factors in Computing Systems (CHI '10). ACM, New York, NY, USA, 2347-2350. DOI: http://dx.doi.org/10.1145/1753326.1753679

[25] Samuel Shaki, Martin Fischer and William Petrusic. 2009. Reading habits for both words and numbers contribute to the snarc effect. Psychonomic Bulletin \& Review 16, 2 (Apr 2009), 328-331

[26] R. William Soukoreff and I. Scott MacKenzie. 2004. Towards a Standard for Pointing Device Evaluation, Perspectives on 27 Years of Fitts' Law Research in HCI. Int. 7. Hum.-Comput. Stud. 61, 6 (Dec. 2004), 751-789. DOI: http://dx.doi.org/10.1016/j.ijhcs.2004.09.001

[27] Sparkfun. 2107. SoftPot Membrane Potentiometer 200mm https://www.sparkfun.com/products/8679. (2017). Accessed: 11-01-2018

[28] Melanie Tory and Robert Kincaid. 2013. Comparing physical, overlay, and touch screen parameter controls. In Proceedings of the 2013 ACM international conference on Interactive tabletops and surfaces (ITS '13). ACM, New York, NY, USA, 91-100. DOI: http://dx.doi.org/10.1145/2512349.2512812

[29] Giovanni Maria Troiano, Esben Warming Pedersen, and Kasper Hornbæk. 2014. User-defined gestures for elastic, deformable displays. In Proceedings of the 2014 International Working Conference on Advanced Visual Interfaces (AVI '14). ACM, New York, NY, USA, 1-8. DOI: https://doi.org/10.1145/2598153.2598184

[30] UK National Health System. Repetitive Strain Injury (RSI) https://www.nhs.uk/conditions/repetitive-strain-injury-rsi/. (2011). Accessed: 11-01-2018

[31] USA Department of Health \& Human Services. System Usability Scale (SUS) https://www.usability.gov/how-to-and-tools/methods/system-usabilityscale.html. (2011). Accessed: 11-01-2018

[32] Jacob O. Wobbrock, Leah Findlater, Darren Gergle, and James J. Higgins. 2011 The Aligned Rank Transform for Nonparametric Factorial Analyses Using Only Anova Procedures. In Proceedings of the SIGCHI Conference on Human Factors in Computing Systems (CHI '11). ACM, New York, NY, USA, 143-146. DOI: http://dx.doi.org/10.1145/1978942.1978963

[33] Pawel Woźniak, Morten Fjeld, and Shengdong Zhao. 2014. Limiting trial and error: introducing a systematic approach to designing clutching. In Proceedings of the Second International Symposium of Chinese CHI (Chinese CHI '14). ACM, New York, NY, USA, 35-39. DOI: http://dx.doi.org/10.1145/2592235.2592241

[34] Bo Yi, Xiang Cao, Morten Fjeld, and Shengdong Zhao. 2012. Exploring user motivations for eyes-free interaction on mobile devices. In Proceedings of the SIGCHI Conference on Human Factors in Computing Systems (CHI '12). ACM, New York, NY, USA, 2789-2792. DOI: http://dx.doi.org/10.1145/2207676.2208678

[35] Neng-Hao Yu, Da-Yuan Huang, Jia-Jyun Hsu, and Yi-Ping Hung. 2013. Rapid selection of hard-to-access targets by thumb on mobile touch-screens. In Proceedings of the 15th international conference on Human-computer interaction with mobile devices and services (MobileHCI '13). ACM, New York, NY, USA, 400-403. DOI: http://dx.doi.org/10.1145/2493190.2493202 